\title{
Simulating and predicting solar cycles using a flux-transport dynamo
}

\author{
Mausumi Dikpati ${ }^{1}$ \\ ${ }^{1}$ High Altitude Observatory, National Center for Atmospheric Research, 3080 Center Green, \\ Boulder, CO 80301, USA \\ email: dikpati@hao.ucar.edu
}

\begin{abstract}
We review how the so-called flux-transport solar dynamos work and show that such models calibrate well with solar cycle observations, and simulate well the relative peaks of the past 8 cycles. This success provides a basis for forecasting a strong solar cycle 24 . We also show that a previous forecast of the timing of onset of cycle 24 is being verified by recent sunspot area statistics and coronal structures.
\end{abstract}

Keywords. Sun: activity, Sun: interior, Sun: magnetic fields, Sun: photosphere, Sun: sunspots

\section{Introduction}

The solar cycle is known to us for 2000 years. The appearance and variations in the number of sunspots with an 11-year periodicity, the reversal of the Sun's polar fields every 11 year, and the variations in the solar corona are the best known manifestations of the Sun's activity cycle. It is widely accepted that a magnetohydrodynamic dynamo produces solar cycle by generating magnetic fields within the Sun.

Observations indicate that the small-scale dynamos, responsible for short-term random magnetic features, and the large-scale dynamo that is responsible for producing the cyclic evolution of global solar magnetic fields, coexist in the Sun. In the present paper, we will focus on large-scale dynamos.

Large-scale solar dynamo models have evolved over the past half a century since the development of the first by Parker (1955). Survey on historical evolution of the large-scale solar dynamo models can be found in recent reviews (Ossendrijver (2003); Dikpati (2004)). We discuss here a widely accepted recent dynamo model, namely a flux-transport dynamo, that have been successful in reproducing many solar cycle features (Wang \& Sheeley (1991); Choudhuri, Schüssler \& Dikpati (1995); Durney (1995); Dikpati \& Charbonneau (1999); Dikpati, de Toma, Gilman, et al. (2004); Rempel (2006)). A flux-transport dynamo is basically a large-scale, $\alpha-\Omega$ dynamo which includes meridional circulation as an important process for transporting magnetic flux via the conveyor belt of plasma flow. Figure 1 illustrates the basic processes involved in a flux-transport dynamo. The meridional circulation plays an important role by determining the dynamo cycle period as well as the memory about the Sun's past magnetic fields in this class of dynamos.

Building on continued successes of kinematic, flux-transport dynamos, we (Dikpati, de Toma \& Gilman (2006); Dikpati \& Gilman (2006)) have recently constructed a fluxtransport dynamo-based predictive tool and applied it to the prediction of cycle 24 . In subsequent sections, we discuss the development of our predictive tool and the results obtained from it. We close by commenting on our future goals. 


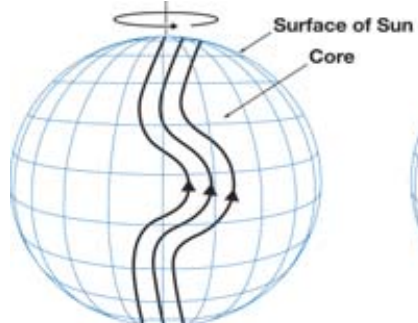

(a)

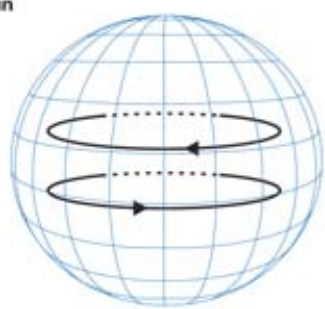

(b)

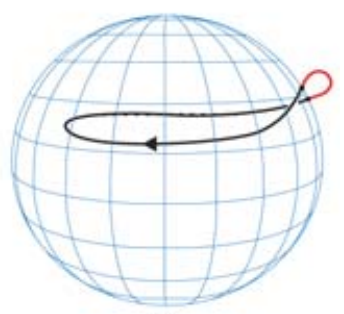

(c)

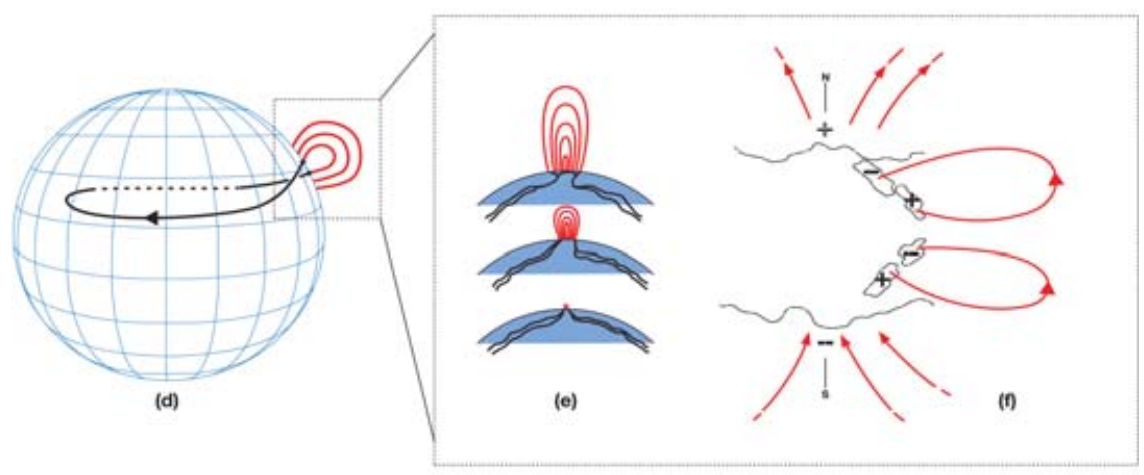

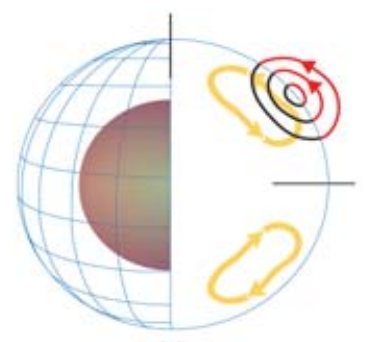

(g)

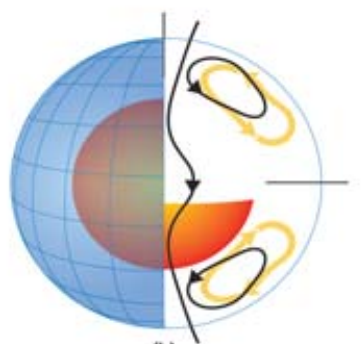

(h)

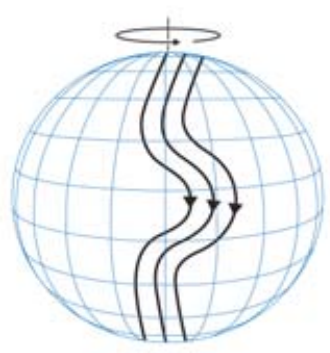

(i)

Figure 1. Schematic of solar flux-transport dynamo processes. Red inner sphere represents the Sun's radiative core and blue mesh the solar surface. In between is solar convection zone where dynamo resides. (a) Shearing of poloidal field by the Sun's differential rotation near convection zone bottom. The Sun rotates faster at the equator than the pole. (b) Toroidal field produced due to this shearing by differential rotation. (c) When toroidal field is strong enough, buoyant loops rise to the surface, twisting as they rise due to rotational influence. Sunspots (two black dots) are formed from these loops. (d,e,f) Additional flux emerges (d,e) and spreads (f) in latitude and longitude from decaying spots (as described in figure 5 of Babcock (1961)). (g) Meridional flow (yellow circulation with arrows) carries surface magnetic flux poleward, causing polar fields to reverse. (h) Some of this flux is then transported downward to the bottom and towards the equator. These poloidal fields have sign opposite to those at the beginning of the sequence, in frame (a). (i) This reversed poloidal flux is then sheared again near the bottom by the differential rotation to produce the new toroidal field opposite in sign to that shown in (b) (adopted from Dikpati \& Gilman (2006)).

\section{Model description}

Starting point of the model is a mean-field, kinematic flux-transport dynamo the equations for which can be written as:

$$
\frac{\partial A}{\partial t}+\frac{1}{r \sin \theta}(\mathbf{u} . \nabla)(r \sin \theta A)=\eta\left(\nabla^{2}-\frac{1}{r^{2} \sin ^{2} \theta}\right) A+S\left(r, \theta, B_{\phi}\right)+\alpha B_{\phi},
$$



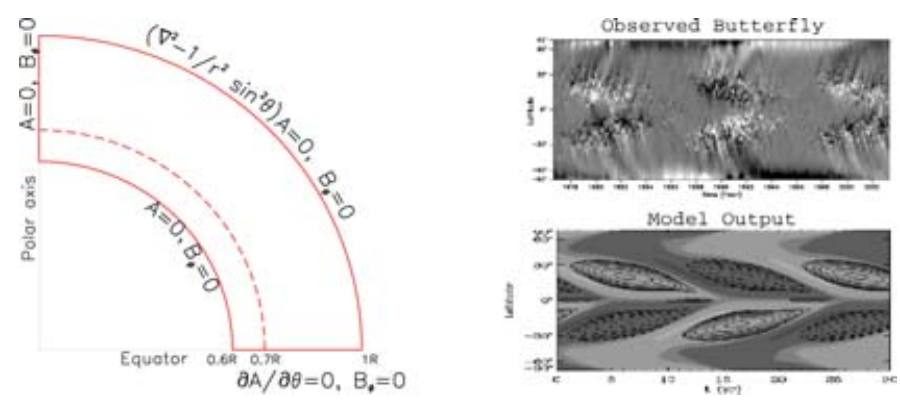

Figure 2. (a) Boundary conditions applied in a single hemisphere calculation; equatorial condition is removed in a full-spherical shell calculation. (b) Top frame plots the observed longitude-averaged photospheric magnetic field data, obtained from Kitt Peak National Solar Observatory, in a time-latitude diagram. Bright(dark) denotes positive(negative) fields. Bottom frame presents a theoretical time-latitude diagram: solid (dash) contours denote toroidal field amplitude at the base of convection zone, and bright (dark) shades the positive (negative) surface radial fields.

$$
\begin{aligned}
\frac{\partial B_{\phi}}{\partial t}+\frac{1}{r}\left[\frac{\partial}{\partial r}\left(r u_{r} B_{\phi}\right)+\frac{\partial}{\partial \theta}\left(u_{\theta} B_{\phi}\right)\right]= & r \sin \theta\left(\mathbf{B}_{p} \cdot \nabla\right) \Omega-\hat{\mathbf{e}}_{\phi} \cdot\left[\nabla \eta \times \nabla \times B_{\phi} \hat{\mathbf{e}}_{\phi}\right] \\
& +\eta\left(\nabla^{2}-\frac{1}{r^{2} \sin ^{2} \theta}\right) B_{\phi},
\end{aligned}
$$

where $\mathbf{B}_{p}=\nabla \times\left(A \hat{\mathbf{e}}_{\phi}\right)$, denotes the poloidal field, $B_{\phi}$ the toroidal field, $\mathbf{u}$ the meridional flow, $\Omega$ the differential rotation, $S\left(r, \theta, B_{\phi}\right)$ the surface poloidal field source-term, and $\alpha$ denotes an $\alpha$-effect arising from the tachocline instability in the overshoot tachocline.

The dynamo ingredients, such as the surface poloidal source, the differential rotation and the meridional circulation, are fully or partially fixed by observations. The only unknown parameter of the model is the diffusivity profile. We solve the equations (1) and (2) numerically under suitable boundary conditions as shown in Figure 2a (for details, see Dikpati \& Charbonneau (1999) and Dikpati \& Gilman (2001)).

We plot in Figure $2 b$ the theoretical time-latitude diagram (bottom frame) derived from the model-output, and compare with the observations (top frame). This model is heavily constrained by observations, without much scope of adjusting the parameters other than the diffusivity; very good agreement of the model-output with observations validates our model.

\section{Application to solar cycle prediction: 2D data assimilation}

The key point in the development of the predictive tool is the assimilation of long-term solar magnetic field data into the model, so that the model builds the memory of the Sun's past magnetic pattern. This is a very similar approach to the data assimilation in atmospheric global circulation models which started about 50 years ago, and has reached a state now that produces the best weather forecasts. In order to apply our flux-transport dynamo model for predicting the mean amplitude and the mean duration of an upcoming solar cycle, we first convert the self-excited dynamo into a forced system by replacing the theoretical Babcock-Leighton poloidal source-term by a forcing term that feeds the model the observed poloidal fields from the surface. The equation (1) is rewritten as:

$$
\frac{\partial A}{\partial t}+\frac{1}{r \sin \theta}(\mathbf{u} . \nabla)(r \sin \theta A)=\eta\left(\nabla^{2}-\frac{1}{r^{2} \sin ^{2} \theta}\right) A+\mathcal{F}(r, \theta, t)+\alpha B_{\phi}
$$




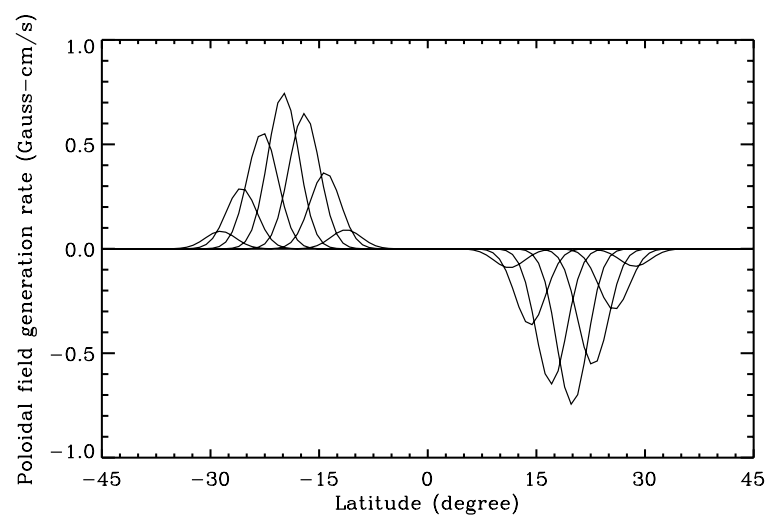

Figure 3. Gaussian band captures the observed surface source pattern migrating from $35^{\circ}$ latitude to the equator during a sunspot cycle. Seven cycle phases are shown. We use each individual cycles amplitudes to determine the height of the Gaussian for all cycle phases (adopted from Dikpati \& Gilman (2006))

The forcing term, $\mathcal{F}$, is derived (see Dikpati \& Gilman 2006, for details) from the observed, longitude-averaged photospheric magnetic flux arising from the decay of active regions. So at a particular time, $\mathcal{F}$ works in a latitude of $6-10^{\circ}$ width, which is represented in Figure 3 by a Gaussian curve. The pattern varies in amplitude and migrates equatorward following the solar cycle.

The time variation of meridional flow not being known prior to 1996, we incorporate here a surface flow speed of $12.5-17 \mathrm{~m} \mathrm{~s}^{-1}$ which is consistent with observations.

By incorporating such poloidal field source since cycle 12 into the model through the aforementioned forcing term, we run the model extending beyond the present up to 2020 and predict that the upcoming cycle 24 will be $30-50 \%$ stronger than the current cycle 23. Figure 4 shows that this result is robust for a wide range of convection zone diffusivity - the only tunable parameter in the model.

In order to extend beyond the present, we have applied three different techniques for deriving the surface poloidal source for the cycle to-be-predicted: (i) assuming the repeat of cycle 23 source, (ii) assuming 'zero' surface source after the present, (iii) deriving a theoretical Babcock-Leighton type surface source from bottom toroidal fields. We find the same answer for the cycle 24 forecast for all three methods, because the cycle 24 amplitude does not depend on that cycle's surface source in this class of model. We will elaborate this point in the next section.

\section{Mechanism of how the model works}

Analysing the animated evolution of magnetic field patterns in our model, we find that the latitudinal components of the poloidal fields from the past three cycles, lined up in the meridional circulation conveyor belt, combine by diffusion to form the 'seed' for the next cycle's spot-producing toroidal field at the bottom of the convection zone. This is in contrast to polar field precursor method, a widely used earlier method (Schatten (2005); Svalgaard, Cliver \& Kamide (2005)), in which the past cycle's radial polar field is responsible for the next cycle's strength. As an example, the Figure 5 shows that the latitudinal fields (top left) recycled back from cycles 16, 17 and 18 combine to produce the 'seed' for cycle 19 (bottom left).

We note that the predictive capability disappears beyond a certain high value of the convection zone diffusivity, namely above $10^{12} \mathrm{~cm}^{2} \mathrm{~s}^{-1}$. For such a high diffusivity case, 

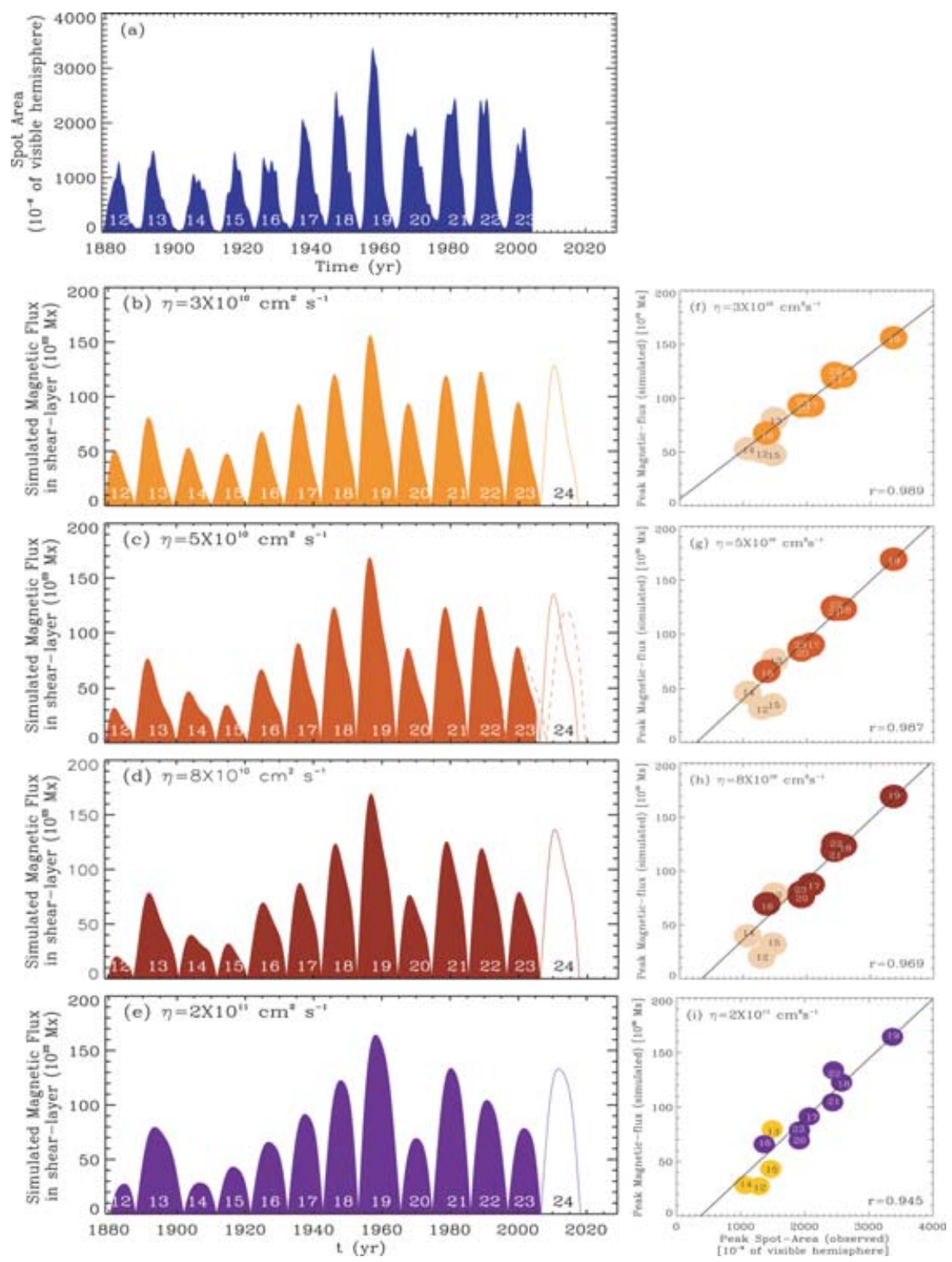

Figure 4. Frame (a) shows observed spot area (smoothed by Gaussian running average over 13 rotations) plotted as function of time. Frames (b,c,d,e) show simulated toroidal magnetic flux in the overshoot tachocline between the equator and $45^{\circ}$ respectively for the cases with convection-zone diffusivity parameters of $3 \times 10^{10} \mathrm{~cm}^{2} \mathrm{~s}^{-1}, 5 \times 10^{10} \mathrm{~cm}^{2} \mathrm{~s}^{-1}, 8 \times 10^{10} \mathrm{~cm}^{2} \mathrm{~s}^{-1}$ and $2 \times 10^{11} \mathrm{~cm}^{2} \mathrm{~s}^{-1}$. Solid red and dashed red curves in frame (c) respectively correspond to the cases with a steady meridional flow and with the time-varying flow incorporated since 1996. Frames (f-i) show correlation plots of simulated cycle peaks vs. observed cycle peaks from spot area for cycles 12 through 23 respectively for the cases as shown in (b-e) (adopted from Dikpati \& Gilman (2006)).

the snapshot of field patterns in Figure 6 shows that the emerged surface poloidal flux from decaying active regions diffuses back down to the bottom in perhaps less than a year. That means that the switching off the surface source for the cycle to be predicted would lead to immediate decay of the next cycle's seed. It also leads to the wrong sign for the next cycle. 

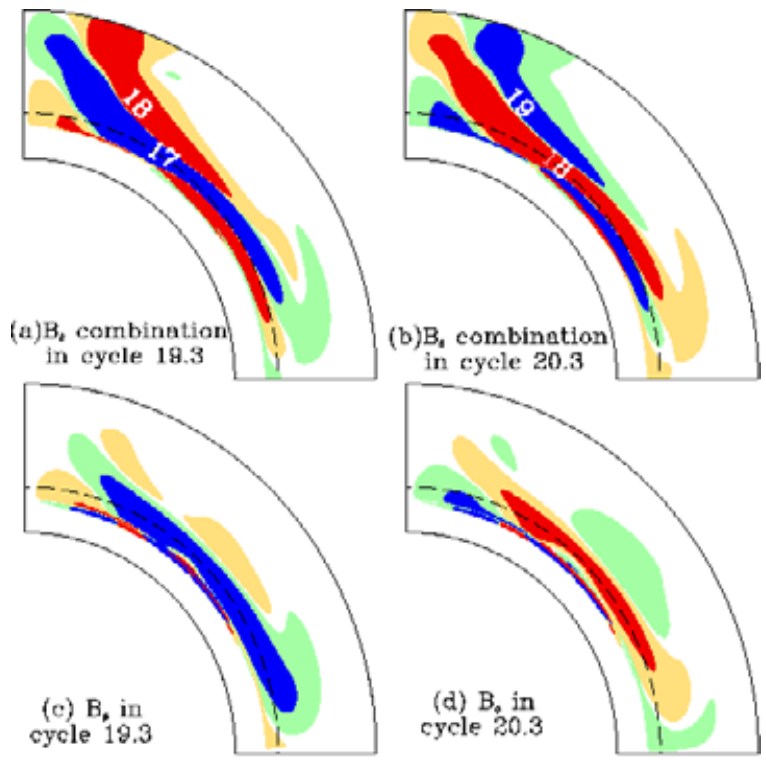

Figure 5. Frames (a) and (b) respectively show the latitudinal component of poloidal field $\left(B_{\theta}\right)$ patterns in cycles 19.3 and 20.3; (c) and (d) show spot-producing toroidal fields generated at convection zone base. In frame (a), $B_{\theta}$ from 3 past cycles $(16,17$ and 18), lined up in meridional circulation conveyor belt, combine to produce the 'seed' for cycle 19.3 toroidal field plotted in frame (c). Similarly $B_{\theta}$ from cycles 17, 18 and 19 in frame (b) combine to produce the 'seed' for cycle 20.3 toroidal field, plotted in frame (d).)

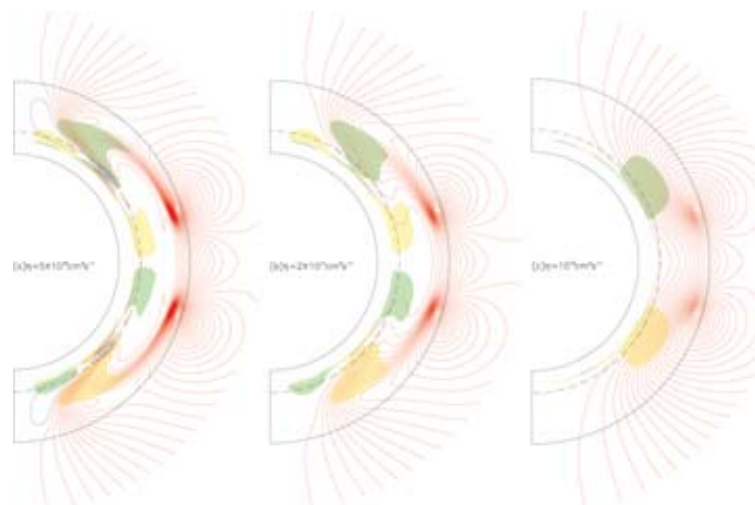

Figure 6. Snapshots of field configurations with toroidal field amplitudes (color shades) and poloidal field lines (contours) for 3 selected convection zone diffusivities noted in frames (a), (b) and (c). Yellow denotes positive toroidal fields that are pointing into the page, and green negative; red (blue) contours denote positive (negative) poloidal field (adopted from Dikpati \& Gilman (2006)).

\section{Discussions}

The flux-transport dynamos driven by the observed surface poloidal source show sginificant skill in predicting the strength of one cycle ahead. Dikpati \& Gilman (2006) have shown that this class of models has certain skill in predicting two cycles ahead - further refinement is necessary to improve the cycle and predict the strength of cycle 25 .

Our forecast of the amplitude of cycle 24 is in good agreement with Hathaway \& Wilson (2004) and Tsirulnik, Kutnetsova \& Oraevsky (1997), but in contrast to that of 

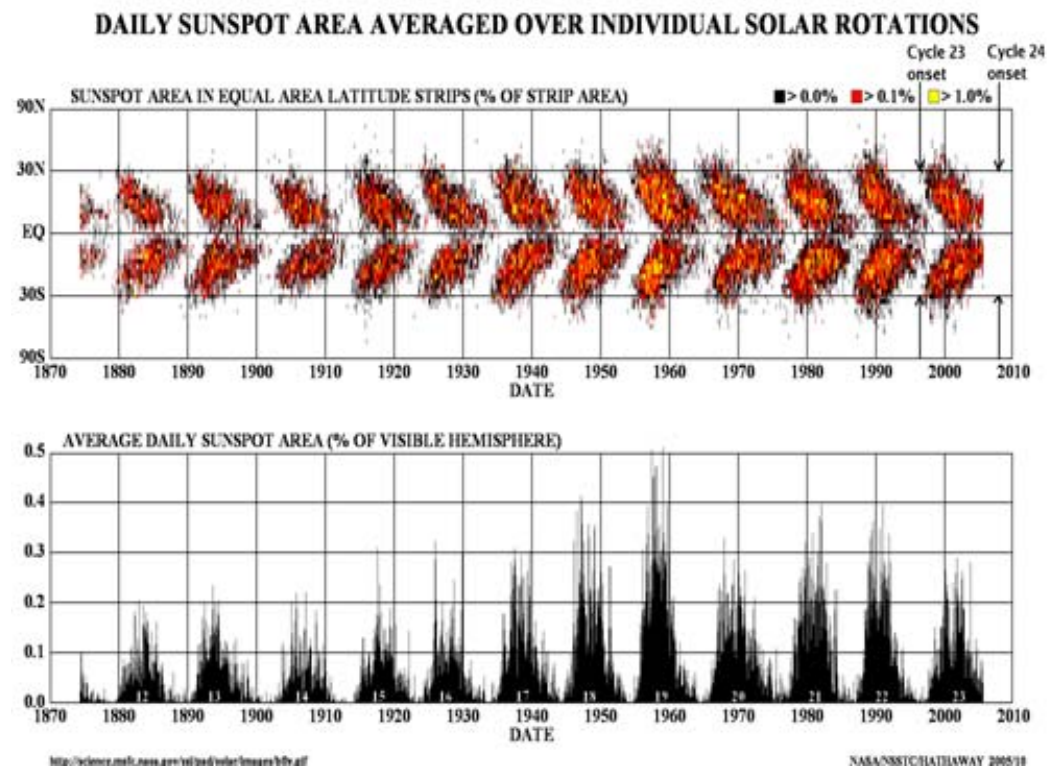

Figure 7. Long-term butterfly diagram taken from Hathaway url. Prejected minimum upcoming at the end of cycle 23 has been marked by arrow.
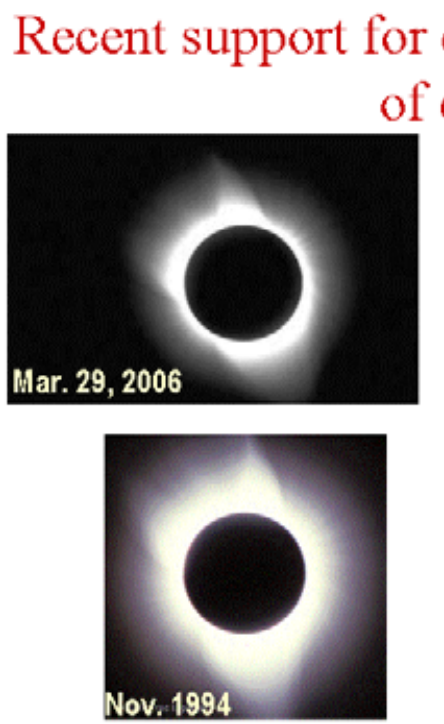

\section{Corona at last solar minimum looked like this}

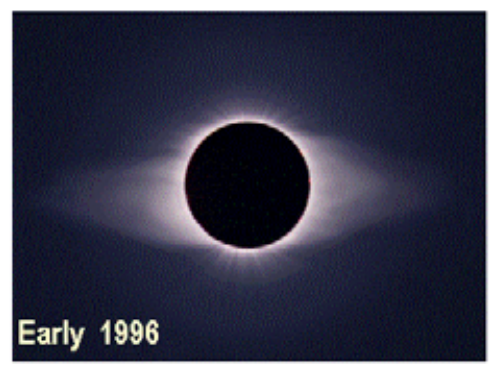

Figure 8. Large scale corona obtained from eclipse at Egypt, March 29, 2006 (top left), from eclipse at Bolivia in November 1994 (bottom left). The solar minimum Corona in early 1996 is at bottom right, for comparison.

Svalgaard, Cliver \& Kamide (2005) and Schatten (2005) who predicted a very low cycle 24 . We have to wait a few years to see whether the various forecasts for cycle 24 verify or not. However, regarding the onset-timing, we note from the long-term butterfly diagram presented in Figure 7, that the current phase of cycle 23 is about 1-1.5 years behind the upcoming solar minimum. The recent large-scale coronal structure also clearly indicates 
that it looks more like November 1994 corona, rather than the solar minimum corona in early 1996 (see Figure 8). The delayed onset of cycle 24 predicted two years ago (Dikpati (2004)) is now supported by these observations. Given such indications about the skill of our model, we will proceed forward to predict the North and South hemispheric solar cycle features separately. We will also assimilate data into the model since cycle 1 so that we can develop some understanding how the Grand minima occurred.

\section{Acknowledgements}

We thank Peter Gilman for reviewing the manuscript and for his helpful comments. We extend our thanks to Eric Priest for raising certain issues on dynamo theory during the IAU Symposium 233 - the answers to which have improved the paper. This work is partially supported by NASA grants NNH05AB521, NNH06AD51I and the NCAR Director's opportunity fund. National Center for Atmospheric Research is sponsored by the National Science Foundation.

\section{References}

Babcock, H.W. 1961, ApJ 133, 572

Choudhuri, A.R., Schüssler, M. \& Dikpati M. 1995, A \& A 303, L29

Dikpati, M. 2004, in: T. Sakurai \& T. Sekii (eds.), The Solar-B Mission and the Forefront of Solar Physics, ASP Conference Series, vol. 325, p. 37

Dikpati, M. 2004, in: D. Dansey (eds.), Proceedings of the SOHO 14/GING 2004 Workshop "Helioseismology and Asteroseismology: Towards a Golden Future", ESA-SP, vol. 559, p. 233

Dikpati, M. \& Charbonneau, P. 1999, ApJ 518, 508

Dikpati, M. \& Gilman, P.A. 2001, ApJ 559, 428

Dikpati, M. \& Gilman, P.A. 2006, ApJ 649, (in press)

Dikpati, M., de Toma, G. \& Gilman P.A. 2006, Geophys. Res. Lett. 33, L05102

Dikpati, M., de Toma, G., Gilman, P.A., Arge, C.N., White, O.R. 2004, ApJ 601, 1136

Durney, B.R. 1995, Sol. Phys. 160, 213

Hathaway, D.H. \& Wilson, R.M. 2004, Sol. Phys. 224, 5

Ossendrijver, M. 2003, Astron. Astrophys. Rev. 11, 287

Parker, E.N. 1955, ApJ 122, 293

Rempel, M. 2006, ApJ 647, (in press)

Schatten, K.H. 2005, Geophys. Res. Lett. 32, L21106

Svalgaard, L., Cliver, E.W. \& Kamide, Y. 2005, Geophys. Res. Lett. 32, L01104

Tsirulnik, L.B., Kutnetsova, T.V. \& Oraevsky, V.N. 1997, Adv. Space Res. 20, 2369

Wang, Y.-M. \& Sheeley, N.R. Jr. 1991, ApJ 375, 761 